\title{
Modeling Neurological Disorders by Human Induced Pluripotent Stem Cells
}

\author{
Tanut Kunkanjanawan, Parinya Noisa, and Rangsun Parnpai \\ Embryo Technology and Stem Cell Research Center, School of Biotechnology, Suranaree University of Technology, \\ 111 University Avenue, Nakhon Ratchasima 30000, Thailand \\ Correspondence should be addressed to Parinya Noisa, p.noisa@sut.ac.th and Rangsun Parnpai, rangsun@g.sut.ac.th
}

Received 11 July 2011; Accepted 6 October 2011

Academic Editor: Ken-ichi Isobe

Copyright ( $\odot 2011$ Tanut Kunkanjanawan et al. This is an open access article distributed under the Creative Commons Attribution License, which permits unrestricted use, distribution, and reproduction in any medium, provided the original work is properly cited.

\begin{abstract}
Studies of human brain development are critical as research on neurological disorders have been progressively advanced. However, understanding the process of neurogenesis through analysis of the early embryo is complicated and limited by a number of factors, including the complexity of the embryos, availability, and ethical constrains. The emerging of human embryonic stem cells (hESCs) and induced pluripotent stem cells (iPSCs) has shed light of a new approach to study both early development and disease pathology. The cells behave as precursors of all embryonic lineages; thus, they allow tracing the history from the root to individual branches of the cell lineage tree. Systems for neural differentiation of hESCs and iPSCs have provided an experimental model that can be used to augment in vitro studies of in vivo brain development. Interestingly, iPSCs derived from patients, containing donor genetic background, have offered a breakthrough approach to study human genetics of neurodegenerative diseases. This paper summarizes the recent reports of the development of iPSCs from patients who suffer from neurological diseases and evaluates the feasibility of iPSCs as a disease model. The benefits and obstacles of iPSC technology are highlighted in order to raising the cautions of misinterpretation prior to further clinical translations.
\end{abstract}

\section{Introduction}

Neurodegenerative and neurodevelopmental diseases are the important causes of disability and death of humans. Although the rapid development of novel diagnostic methods and therapeutic approaches has been in progress, there is no emergence of an efficient way to prevent and cure these diseases. Due to the lack of a suitable disease model and an adequate number of biopsy brain samples, the genuine etiology and pathology of many nervous diseases are unidentified. Transgenic animals for disease modeling were developed, and many of them show disease pathology and response to treatment trials. For example, the Alzheimer's disease (AD) mice, which overexpress familial type AD-associated genes, amyloid precursor protein (APP), Presenilin1/2 (PS), and Tau, show clinical pathologies of $\mathrm{AD}$, such as progressive memory loss, extracellular plaque, and neurofibrillary tangles [1]. Although animal model continues to produce key insights into disease mechanisms, these systems have limitations that could be potentially overcome by human cellular models of diseases. Many transgenic murines do not faithfully mirror the respective human pathophysiology. For example, a mouse model for Down syndrome (DS) fails to recapitulate the human cranial abnormalities, a common associated feature of trisomy 21 [2]. This may suggest that the mouse model for human trisomy 21 is not fully appropriate, and an alternative system is necessary for exploring disease mechanisms [3].

Human embryonic stem cells (hESCs) are isolated from the inner cells mass of blastocyst stage embryos which can be further differentiated into three embryonic germ layers: ectoderm, mesoderm, and endoderm [4]. Recent results indicate that the differentiation of hESCs in culture follows the hierarchical sets of signals that regulate embryonic development in the generation of the germ layers and specific cell types [5]. Establishment of in vitro differentiation systems that recapitulate normal development will form the foundation for dissecting molecular interactions. The ability 
to access and manipulate populations representing early developmental stages in the hESC differentiation cultures provides a new approach for addressing questions of lineage commitment, such as neurodevelopment [6]. This system provides a model of human brain development that enables manipulations comparable to those carried out in other organisms such as Xenopus and zebrafish, but in human species context. In order to derive disease-specific hESCs, there are two conventional methods which are (1) the isolation of single blastomere from morula stage embryos which are entered into preimplantation genetic diagnostic (PGD) program and (2) the derivation of hESCs by somatic cell nuclear transfer (SCNT) [7]. PGD is a clinical procedure for screening fertilized embryos at morula stage in order to ensure disease-free embryos [8]. The embryos that are diagnosed as disease threatened will be either discarded or donated for research [9]. On the other hand, SCNT is an alternative approach to generate patient-specific hESCs. The nucleus from the somatic donor cells is transplanted into the enucleated oocytes by micromanipulator, leading to union of both components [10]. However, the success rate of hESC establishment form PGD and SCNT is considerably low and technicaly demanding. Moreover, SCNT is limited by the lack of oocyte donors and ethical issues; causing genomic reprogramming by nuclear transfer has not been extensively demonstrated in human [11-13].

Because of the multiple drawbacks of hESCs and the derivation approaches, another pluripotent cells could be derived from somatic cells by the forced expression of key pluripotent transcription factors of hESCs (OCT4, c-MYC, SOX2, and KLF4), and these cells were named as induced pluripotent stem cells (iPSCs) [14, 15]. After the discovery of iPSCs by Takahashi et al. in 2007 , the trend of disease modeling has intensively focused on iPSC technology as this technique could generate pluripotent cells from diagnosed living patients and be further differentiated into disease-relevant cell types for drugs screening and disease development monitoring [16]. Thus, this paper will discuss the current success of iPSC derivation from neurological disease patients which will ultimately lead to an answer of pathological causes and a novel pharmaceutical product to treat those diseases.

\section{Insights: The Properties and Origins of Human Pluripotent Stem Cells}

Primarily, hiPSCs have been characterized by following the characteristics of hESCs. These two pluripotent cell types display several similar properties, such as their morphology, self-renewal, differentiation capacity, cell surface antigens, and gene expression profile [16]. They are immortalized cells and could be differentiated into all primary embryonic germ layers, including gut epithelium, cartilage, bone, smooth muscle, striated muscle, neural epithelium, embryonic ganglia, and stratified squamous epithelium [17]. hESCs and iPSCs show the high expression of telomerase reverse transcriptase [18] as well as the stage specific embryonic antigens (SSEAs), which are SSEA-3, SSEA-4, TRA-1-60 and TRA-1-81. Interestingly, it was demonstrated that the expression pattern of these surface antigens is present in the ICM of human blastocysts, providing evidence that hESCs continue to resemble the cells in the ICM [19]. Self-renewal and pluripotency of hESCs and iPSCs are mainly controlled by intrinsic transcription factors. The best-studied intrinsic factors are Oct4, Nanog, and Sox2, which play essential roles in both mESCs and hESCs. The downregulation of these factors leads to hESC differentiation [20-22]. The significant roles of these transcription factors in pluripotency have been confirmed by the ability to reprogram human fibroblasts to become pluripotent cells [16]. As exhibiting pluripotency, when embryoid body is formed from hESCs and iPSCs, the cell aggregates show similar structure to early stage of human embryos which consist of all three embryonic germ layers [23]. hESCs can also form teratomas following the injection of cells into nude mice, which reflects their in vivo differentiation capability $[24,25]$. Even if hESCs have been considered a cell resource for regenerative medicine, there are several controversial issues needed to be taken into account, including ethical constrains of embryo destruction, graftversus-host disease, and the difficulty to obtain diseasedspecific cell lines. Since the discovery of iPSCs by using dermal fibroblasts, scientists have extended to various origins of starting cells such as neural stem cells [26], adiposederived mesenchymal stem cells [27], umbilical cord blood [28], and T-cell lymphocyte [29, 30]. For these reasons, iPSCs are suggested to become a new paradigm for generating patient-specific pluripotent stem cells to model neurological genetic diseases.

\section{3. hiPSCs Breakthrough: The Systems for Neurological Disease Modeling}

In clinical research, it is hard to obtain brain tissues from either live or dead patients for investigating diseases. This difficulty has limited the knowledge of human neurological abnormalities and pathology progression during the course of diseases [31]. Reprogramming of somatic cells from nervous disorder patients by using iPSC technology has provided an opportunity to generate disease-harboring pluripotent cells which can be differentiated into neural cells for studying disease development. Dermal fibroblast cells are widely used as starting cells for somatic cell nuclear reprogramming. The differentiation propensity of iPSCs toward specific cell types, such as neural cells, is marked by the epigenetic memory of starting cells [32]. The current established iPSCs derived from neurodevelopment and neurodegenerative disorder patients are summarized in Table 1, while the similarities and differences of ESCs and iPSCs are illustrated in Table 2. Categorizing by types of diseases, neurological disease-specific iPSCs could be divided into two major groups which are early-onset neurodevelopment diseases and late-onset neurodegenerative diseases. Neurodevelopment diseases are mostly triggered by abnormal gene expression, while neurodegenerative diseases typically resulted from both abnormal gene expression and environment factors. It is an advantage of iPSCs to mimic neurodevelopment disorders 
TABLE 1: List of reported hiPSC disease models.

\begin{tabular}{|c|c|c|c|c|c|}
\hline \multirow{2}{*}{ Disease } & \multirow{2}{*}{ Target cell } & \multicolumn{2}{|c|}{ Potential to be disease model } & \multirow{2}{*}{ Drug test } & \multirow{2}{*}{ Reference } \\
\hline & & $\begin{array}{l}\text { Successful differentiated } \\
\text { into target cell type }\end{array}$ & Neuronal pathology & & \\
\hline \multicolumn{6}{|c|}{ Early-onset neurological disorders } \\
\hline Fragile X syndrome & ND & ND & $\begin{array}{l}\text { Loss of FMR1 } \\
\text { expression }\end{array}$ & ND & [44] \\
\hline Prader-Willi syndrome & Neurons & Yes & Imprint disorder & ND & {$[48,65]$} \\
\hline Rett's syndrome & Neurons & Yes & $\begin{array}{l}\text { Loss of synapses, } \\
\text { reduced spine density, } \\
\text { smaller soma size }\end{array}$ & Yes & {$[31]$} \\
\hline Familial dysautonomia & Neural crest cells & Yes & Loss of neural crest cells & Yes & [54] \\
\hline Friedreich's ataxaia & Motor neuron & Yes & $F X N$ gene repression & ND & {$[61]$} \\
\hline Angelman's syndrome & Neurons & Yes & Imprint disorder & ND & {$[65]$} \\
\hline Down's syndrome & Neuron & ND & ND & ND & [69] \\
\hline Spinal muscular atrophy & Motor neurons & Yes & $\begin{array}{l}\text { Loss of neuron } \\
\text { formation, loss of } S M N \\
\text { gene expression }\end{array}$ & Yes & {$[34]$} \\
\hline \multicolumn{6}{|c|}{ Late-onset neurological disorders } \\
\hline $\begin{array}{l}\text { Amyotrophic lateral } \\
\text { sclerosis (ALS) }\end{array}$ & Motor neurons & ND & Not shown & ND & {$[38]$} \\
\hline Huntington's disease (HD) & Striatal neurons & Yes & Not shown & ND & [37] \\
\hline Parkinson's disease $(\mathrm{PD})$ & $\begin{array}{l}\text { Dopaminergic } \\
\text { neurons }\end{array}$ & Yes & Not shown & ND & {$[35,36]$} \\
\hline Alzheimer's disease $(\mathrm{AD})$ & $\begin{array}{l}\text { Cholinergic } \\
\text { neurons }\end{array}$ & Yes & $\begin{array}{l}\text { Increase ratio of } A \beta 42 \\
\text { to } A \beta 40\end{array}$ & Yes & {$[87]$} \\
\hline
\end{tabular}

${ }^{*} \mathrm{ND}$ : not determined.

TABLE 2: Summarizing the similarities and differences of ESC and iPSC for disease modeling.

\begin{tabular}{|c|c|c|c|}
\hline & hESCs & iPSCs & Reference \\
\hline Source & ICM & Adult somatic cell & {$[4,15]$} \\
\hline \multicolumn{4}{|l|}{ Basic pluripotent characteristics } \\
\hline Alkaline phosphatase activity & Yes & Yes & {$[4,15]$} \\
\hline Pluripotent genes expression & Yes & Yes & {$[4,15]$} \\
\hline Pluripotent cell surface markers & Yes & Yes & {$[4,15]$} \\
\hline In vitro and in vivo multilinage differentiation & Yes & Yes & {$[4,15]$} \\
\hline Transcriptomic expression & Normal pluripotent gene expression & $\begin{array}{l}\text { Pluripotent gene expression } \\
\text { but not all genes similar to } \\
\text { hESCs and depend on } \\
\text { reprogramming technique }\end{array}$ & {$[36,100]$} \\
\hline Proteomic expression & Normal proteomic expression & $\begin{array}{l}\text { Not all proteomic } \\
\text { expression identical to } \\
\text { hESCs }\end{array}$ & {$[101]$} \\
\hline Disease modeling & From PGD diagnosed embryo in IVF clinic & $\begin{array}{l}\text { From diagnosed adult } \\
\text { patient somatic cell }\end{array}$ & {$[88,102]$} \\
\hline Disease-related expression characteristic & Yes & Yes & {$[88,102]$} \\
\hline
\end{tabular}

since neural cells derived from iPSCs display an early stage of disease development [31,33]. However, immature phenotypes of neurons derived from iPSCs hinder the applications of modeling for late-onset diseases [33]. It is important to note that although some neurons derived from iPSCs predominantly exhibited disease pathology and responded to pharmaceutical agents $[31,34,35]$, a number of neural cells from certain diseased iPSCs did not show neuronal pathology, such as Parkinson's disease [35, 36], Huntington's disease [37], and amyotrophic lateral sclerosis [38]. Specific gene mutations or environmental stress inducers are needed in order to accelerate the pathology of those diseases $[35,36]$. 


\section{The Current Available iPSCs from Monogenic Early-Onset Neurological Disorders}

4.1. Fragile $X$ Syndrome. Fragile $X(\mathrm{FX})$ syndrome is an $\mathrm{X}$-linked dominant disorder which is the most common form of inherited mental retardation [39]. The cognitive, behavioral, and physical phenotypes vary by sex in which males are severely affected due to the X-linked inheritance of mutation [40]. This disease caused by no expression of the fragile $\mathrm{X}$ mental retardation 1 (FMR1) gene, resulting from untranslation of CGG triplet repeat expansion in the 5' UTR region of the gene [41]. According to the loss of FMR1 protein, developmental retardation is significantly found in the cerebral cortex. Quantitative examination of human brain autopsy exhibits abnormal dendritic spine lengths, and shapes are more immature when compared with normal age match controls $[42,43]$. Fragile X syndrome iPSCs (FX-iPSCs) were generated from 3 FX-affected males and compared the regulation of FMR1 transcription to human FX-ESCs. FX-iPSCs cloned demonstrated typical characteristic of pluripotent stem cells [44]. Surprisingly, although the mutant FMR1 gene is expressed in FX-ESCs, FMR1 gene expression remains inactive in FX-iPSCs. In addition, even somatic FX-fibroblasts were pretreated with demethylating agent 5-azacytidine prior to reprogramming; FMR1 gene remained transcriptionally silent in all FX-iPSCs clones. This hypothesized that other epigenetic mechanisms may affect the aberrant expression of FMR1 gene. This brought the attention that the differentiation of FX-iPSCs into neurons may not facilitate pathological study of fragile $\mathrm{X}$ syndrome [44]. Further examination of multiple factors, such as epigenetic factors, is required in order to improve FXiPSC properties, in particular gene expression pattern [33].

4.2. Prader-Willi Syndrome (PWS). Prader-Willi syndrome (PWS) is a neurological disorder characterized by neonatal hypotonia, failure to thrive, hypogonadism and short stature, mild-to-moderate mental retardation, and compulsive hyperphagia in early childhood that leads to morbid obesity [45]. PWS and AS are closely related in which the imprinted genes on the proximal long arm of chromosome 15 are affected. PWS is affected by the lack of gene expression in paternal chromosome, 15q11-q13, while genes in maternal chromosome are repressed by DNA methylation [46]. The definition of etiology of this disease is unclear, but a deficiency of the paternally expressed SNORD116 snoRNAs can result in a PWS or PWS-like phenotypes [47]. PWSiPSCs were generated from fibroblast of diagnosed PWS patients by using retrovirus producing 4 exogenous transcription factors. PWS-iPSCs showed positive-to-standard hESC characteristics: cell surface antigens, endogenous hESC transcription factors, and teratoma formation. Moreover, the methylation status of proximal promoter, OCT4 and NANOG, displayed extensive DNA demethylation in PWS iPSCs clone at a comparable level to hESCs. PWS-iPSCs displayed a normal number of chromosomes, but DNA segment translocation was observed from chromosome 15 to chromosome $4[46,48]$. The expression of SNORD116 analyzed by quantitative PCR demonstrated that the high expression of SNORD116 was observed in normal fibroblast and normal iPSCs, but PWS fibroblasts and PWS-iPSCs showed low expression [48]. It is suggested that PWS-iPSCs did not display normal expression pattern of imprinted genes which are crucial for disease determination [48]. The results supported the hypothesis that genomic imprinting is not susceptible to nuclear reprogramming and refractory to acquired de novo alteration.

4.3. Rett's Syndrome. Rett's syndrome is an inherited neurological developmental disorder which is associated with $\mathrm{X}$-linked gene inheritance encoding methyl-CpG-binding protein 2 (MeCP2) [49]. MeCP2 is located in the nucleus of many types of CNS neurons and functions as a transcriptional repressor by associating with chromatin remodeling complexes [50]. This disease is characterized by a variety of clinical manifestations, indicating developmental arrest and psychomotor repression. The patients have mental retardation, epilepsy, respiratory dysfunction, stereotypic hand movement, growth retardation, scoliosis, and spasticity [51]. RTT-iPSCs were generated from four distinct MeCP2 mutation fibroblasts, using retroviral reprogramming vectors. The resulting cells were pluripotent as WT-iPSCs. Immunocytochemistry results against trimethylated histone 3 lysine 27 ( $\mathrm{m} 3 \mathrm{H} 3 \mathrm{~K} 27)$ were positively marked at the nucleus of some, but not all, undifferentiated RTT-iPSCs, similar to the control hESCs. This showed that the memory of the previous chromatin inactivation state had been erased. By immunostaining, RTT fibroblasts and RTT-iPSCs-derived neuronal population reduced $\mathrm{MeCP} 2$ protein levels. Moreover, the half reduction of $\mathrm{MeCP} 2$ protein is consistent with the random $\mathrm{X}$-inactivation. It was noted that $\mathrm{X}$-inactivation was reset in RTT-iPSCs and restored randomly during neuronal differentiation. This caused the variation of MeCP2 protein level, reminiscent of the brain of RTT patients [31]. After 8 weeks of neural differentiation, the number of VGLUT-positive glutamatergic neurons from RTT-iPSCs was less than that derived from either to WT-iPSCs or hESCs. This phenotype could be found when the WT-iPSCs knocked down the expression of MeCP2, while the overexpression of MeCP2 could increase the number of VGLUT1-positive neurons derived from RTT-iPSCs. Morphological analysis of RTT neurons demonstrated that the number of spine of RTT neuritis and cell soma sizes was reduced when compared to WT neurons. Interestingly, high concentration of aminoglycoside antibiotic, such as gentamicin, could increase MeCP2 protein and, consequently, the number of glutamatergic neurons [31]. This result suggested that RTT-iPSCs were providing the excellent disease modeling for RETT syndrome and could confirm the pathology of the disease.

4.4. Familial Dysautonomia. Familial dysautonomia (FD) or Riley Day Syndrome is an autosomal recessive disorder, characterized by the developmental loss of neurons from the sensory and autonomic nervous system $[49,50]$. FD is caused by the mutation in a splice site of the I-kB kinase complex-associated protein (IKBKAP) gene, which causes 
tissue-specific exon skipping and expression of a truncated mRNA transcript [52]. Reduced levels of normal IKAP protein are associated with a defect in cell motility [53]. FDiPSCs were generated from 10-year-old female FD patients using lentiviral vector encoding 4 classical vectors [16]. Genetic defect, homozygous $2507+6 \mathrm{~T}>\mathrm{C}$, was confirmed in FD-iPSCs by sequencing and IKBKAP analysis using RT-PCR. Gene expression profile of neural crest derived from FD-iPSCs showed that, among the 20 most deceased transcripts in FD neural crest precursors, many genes were involved in peripheral neurogenesis and neural differentiation. Moreover, the number of paxillin-positive cells was reduced in FD-iPSCs-derived neural crest progenitors, referring to the aberration of cell spreading and migration [54]. They have reported that plant hormone kinetin could reduce the levels of the mutant IKBKAP splice in FD cells [54-56]. Epigallocatechin gallate and tocotrinal were exposed to FD-iPSCs-derived neural crest precursors and showed dramatic reduction of the mutant IKBKAP splice form; however, the hormone did not show a significant increase in the expression of neurogenic markers or improve the migration behavior [54].

4.5. Friedreich's Ataxia. Friedreich's ataxia (FRDA), the most common inherited ataxia, is associated with a mutation of the frataxin gene on chromosome $9[57,58]$. The incidence of mutations was often found in an unstable expansion of GAA repeats in the first intron. The level of GAA repeats is correlated with the downregulation of gene expression and the progression of disease severity [58]. The cardinal features of FRDA are ataxia of all four limbs, cerebella dysarthria, lack of reflexes, sensory loss, and pyramidal signs. In addition, frataxin insufficiency leaded to skeletal deformities, cardiomyopathy, and the risk of diabetes [57, 58]. Although mice models of this disease are available, they do not fully recapitulate gene silencing and frataxin protein level $[59,60]$. FRDA-iPSCs were established by retroviral transduction with 4 classical transcription factors [61] and showed typical characteristics of pluripotent cells as well as retaining the marked repression of FXN mRNA. FRDA-iPSCs still repeated the GAA expansion in both parental pathogenic alleles. Furthermore, GAA repeat lengths in FRDA-iPSCs change overtime in culture. The role of mismatch repair (MMR) enzyme MSH2 was investigated in FRDA-iPSCs. mRNA expression and western blotting analysis of FRDAiPSCs showed large increases in MSH2 level when compared with donor fibroblast. The FRDA-iPSCs have been expected to provide a valuable modeling to study repeat instability mechanism [61]. Differentiations to disease-specific cell types, such as neurons, cardiomyocytes, and pancreatic beta cells, for studing effect of abnormality are required. Moreover, the novel drugs or therapeutic protocols are expected to develop by using this FRDA-iPSCs modeling.

4.6. Angelman's Syndrome. Angelman's syndrome (AS) is clinically delineated by the combination of seizure, absent speech, and hypermotoric and ataxic movements $[62,63]$. AS patients exhibit a predisposition toward apparent happiness and paroxysms of laughter, and this finding helps to distinguish AS from others involving severe developmental handicap [63]. AS is a severe genetic disorder, caused by mutation or deletion of the maternally inherited UBE3A gene in chromosome 15. This gene encodes an HECT (homologous to E6-associated protein $\mathrm{C}$ terminal) domain $\mathrm{E} 3$ ubiquitin ligase [62]. The combination of epigenetic silencing of paternal allele and gene inactivation of maternal allele of UBE3A leads to chiefly incomplete loss of UBE3A protein selectively in most neurons in the brain [64]. Recently, AS-iPSCs lines were established from fibroblasts of two AS patients, who carried maternally inherited deletions of chromosome 15q11-q13 [65]. The methylation imprinting was assessed and showed similar patterns to patient's fibroblasts. Only an unmethylated paternal allele was observed in AS-iPSCs. Neurons and astrocytes derived from AS-iPSCs were matured in vitro and exhibited train of action potential and excitatory postsynaptic current. The levels of UBE3A expression were significantly reduced in both of AS-iPSCs- and AS-iPSCsderived neurons, while normal iPSCs or iPSCs-derived neurons maintain the level of UBE3A. There is evidence suggesting that brain-specific $U B E 3 A$ repression is mediated by a snoRNA $[47,66,67]$. Northern blot hybridization used to assess expression of snoRNAs, SNORD 116 and SNORD 115, demonstrated that SNORD 116 is expressed in both iPSCs and iPSC-derived neurons derived from normal or AS individuals, whereas SNORD115 expression is restricted to iPSC-derived neurons. It is suggested that the neuronspecific repression of $U B E 3 A$ may occur relatively late during neurogenesis, coincident with the upregulation of SNORD 116 and SNORD115 during neural differentiation. This finding proposed that the methylation imprinting is difficult to be reprogrammed, and the epigenetic status is resistant to a global erasure [65]. This AS-iPSC model recapitulates the tissue-specific pattern of UBE3A imprinting; thus, it provides an important tool to address the timing and mechanisms controlling epigenetic status of UBE3A during human neural development. In addition, AS-iPSCs-derived neurons will also be a useful system for the characterization of the physiological abnormalities of the disease at a cellular level [65].

4.7. Down's Syndrome. Down's syndrome (DS) is a developmental disorder, caused by trisomy of chromosome 21 . The key manifestations of the disease are mental retardation, craniofacial abnormalities, and clinical defections of several systems such as heart, gut, and immune system [68]. However, the trisomy of chromosome 21 in mice did not result in disease symptoms which means that mouse is not a suitable system to model AD pathology [2]. DS-iPSCs were established from DS patient fibroblasts by using either four (Oct4, SOX2, KLF4, and c-MYC), or three (without $c-M Y C$ ) reprogramming factors. DS-iPSCs showed the characteristics of trisomy 21 anomaly by chromosomal G-banding analysis, but none of differentiation studies have been conducted [69]. DS-iPSCs are not only attractive for the investigation of DS development, but also interesting for other DS-related diseases such as $\mathrm{AD}$, which is a frequently coincident disease in DS patients. 
4.8. Spinal Muscular Atrophy. Spinal muscular atrophy (SMA), an autosomal recessive disease, is one of the leading genetic causes of infant mortality due to the specific loss of alpha motor neurons in the spinal cord [70, 71]. Clinically, SMA is caused by the homozygous deletion of survivor motor neuron 1 and 2 (SMN1 and SMN2), and the disease severity spans a broad spectrum, based on the onset period [71]. SMN2-derived pre-mRNA transcripts could be alternatively spliced, causing a single nucleotide difference, a silent cytosine-to-thymine (C-T) transition within exon 7 , from the normal mRNA. This single nucleotide change restricts the length of SMN2 protein and translates to a dysfunction protein, named SMN $\Delta 7$. Patients with high copy number of SMN2 producing more full length of SMN by $S M N 2$, are observed in milder form of the disease [72]. SMA-iPSCs were established from a type I SMA patient and his unaffected mother. iPSCs were generated from primary fibroblasts with lentiviral constructs encoding Oct4, SOX2, NANOG, and LIN28. SMA-iPSCs showed pluripotent characteristics like hESCs and were not different from WTiPSCs. iPSC and fibroblast SMN RNA were analyzed. RTPCR analysis showed that SMA-iPSCs and SMN fibroblast have lower levels of SMN RNA than WT-iPSCs and WT fibroblast. Moreover, qRT-PCR result confirmed the significantly reduced level of full-length SMN transcript in SMAiPSCs, 32-39\% reduction compared to wild type. SMAiPSCs were differentiated into motor neurons which are pathological specific cell types. Interestingly, motor neurons derived from SMA-iPSCs group showed significantly fewer number and reduced size than motor neurons derived from WT-iPSCs. However, there was no difference in total number of Tuj1-positive neurons in either WT or SMA groups. This result suggested that SMA has a specific influence on motor neuron, and the disease phenotype selectively hinders motor neuron production and/or increases motor neuron degeneration at later time point. Neuron and astrocytes derived from SMA-iPSCs significantly increased the level of intranuclear gems, intranuclear form of aggregated SMN protein, after valproic acid and tobramycin, when compared to untreated group [34]. The results indicated that neural cells derived from SMA-iPSCs responded to drug screening and are able to be used as the disease model for further disease investigation.

\section{The Established iPSCs for the Polygenic Late-Onset Neurological Disorders}

5.1. Amyotrophic Lateral Sclerosis. Amyotrophic lateral sclerosis (ALS) is a progressive disease, characterized by the degeneration of upper, in layer $\mathrm{V}$ of the motor cortex, and lower motor neurons, brain stem, and anterior horn of spinal cord. The loss of motor neurons, especially in spinal cord, leads to progressive paralysis and denervation atrophy of striated muscles [73]. Inheritance in familial ALS (FALS) is typically autosomal dominant [74]. The mutation in only one gene, named $\mathrm{Cu} / \mathrm{Zn}$ superoxide dismutase (SOD1), results in classical inherited ALS [75]. iPSCs were developed by using skin fibroblasts from 82- and 89-year-old FALS patients, who are heterozygous of the rare L144F $\left(\mathrm{Leu}^{144} \rightarrow \mathrm{Phe}\right.$ ) dominant allele of SOD1. FALS-iPSCs showed normal characteristic of pluripotent stem cell like hESCs. FALS-iPSCs were differentiated to mature motor neurons, HB9 and ISLET1/2-positive cells, astrocytes, and GFAP-positive cells. The abnormality of motor neurons and differentiated cells from FALS-iPSCs has been described [38]. Thus, the expressions of disease characteristics as well as the pathological anomaly of motor neuron needed further investigations, such as persistent of SOD1 mutation in developed iPSCs.

5.2. Huntington's Disease. Huntington's disease (HD) is characterized by the loss of brain striatal neurons that results from the expansion of a CAG repeat, translated into glutamine and produced mutant huntingtin protein [76]. The pathological change in HD brain is evident by the massive loss of medium spiny neurons (MSNs) in the striatum and loss of neurons in the cortex which results in chorea, dementia, and eventually death [77]. HD-iPSCs line was made from HD patients with 72 repeat CAG nucleotides [37, 69]. Every cell derived from HD-iPSCs, including HD-NSCs and striatal differentiated neurons, contained 72 CAG repeats. HD neural stem cells (HD-NSCs) and striatal neurons could be generated from these HD-iPSCs, but the population of striatal neurons, DARPP-32-positive cells, were dramatically low, approximately $10 \%$ of the total neurons. Caspase activity was evaluated after the withdrawal of growth factors for 24 hours. In HD-NSCs, caspase 3/7 activity was stimulated, but not in WT-NSCs [37]. This suggested that HD-iPSCs and their differentiated striatal neurons are a suitable model for $\mathrm{HD}$, and this could be further supported by the comparison with another alternative system in nonhuman primate ESCs [78]. However, the level of huntingtin protein in HD-iPSCs has not yet been demonstrated.

5.3. Parkinson's Disease. Parkinson's disease is the second most common neurodegenerative disease after Alzheimer's disease [79]. Currently, there have been identified 6 causative genes in which their mutations are associated with the high incidence of PD. These six genes include $\alpha$-synuclein, parkin, UCH-LI, PINK1, DJ-1, and LRRK2/dardarin [80]. The manifestations of PD are resting tremor, bradykinesia, rigidity, and gait impairment. The motion difficulty of $\mathrm{PD}$ patients is attributed to the loss of dopaminergic (DA) neurons within the substantia nigra (SN), causing the dysfunction of the basal ganglia. The motor symptoms and survival rate of PD patients could be relieved by synthetic dopamine replacement [81]. PD-iPSCs were generated from idiopathic PD patients with either 3 (without c-Myc) or 4 transcription factors by using lentivirus flanked with loxP sites. Thus, these exogenous genes could be later removed by Cre recombinase in order to fabricate the factor-free PDiPSCs. Not only establishing the pluripotent state, the factorfree PD-iPSCs also showed a close global gene expression profile to hESCs. DA neurons could be generated from PDiPSCs at a comparable efficiency to non-PD-iPSCs or hESCs [36]. This was suggested due to the short time span of cultured neurons since the onset period of PD patients is approximately over 50 years old. In order to accelerate the 
disease pathology, the exogenous stimuli, such as an exposure to oxidative stress, neurotoxin, or overexpression of PDrelated genes ( $\alpha$-synuclein or LRRK2), may be needed to supplement the culture systems [36]. On the other hand, fibroblasts from monogenic mutation of PD were also reprogrammed to iPSC state. These fibroblasts carried p.G2019S mutation (called G2019S-iPSCs) in the leucine-rich repeat kinese-2 (LRRK2) which is the most common PD-related mutation [35]. DA neurons derived from G2019S-iPSCs showed not only the increased expression of key oxidative stress-response gene and $\alpha$-synuclein protein, but also the high sensitivity to caspase 3 activation and cell-death-causing agents. Moreover, the treatment of cell death inhibitor, ROCK inhibitor, or Y-27632, did not protect G2019S-iPSCderived DA neurons from hydrogen peroxide or MG-132mediated caspase 3 activation [35]. The results emphasized the possibility to generate the late-onset neurodegenerative disease models by using disease-related exogenous stimuli and mutant cell lines. However, the solid protocol of diseasespecific cell type differentiation and long period maintaining differentiated cell in vitro are needed to develop further investigation.

5.4. Alzheimer's Disease (AD). Alzheimer's disease (AD) is known as the most common neurodegenerative disorder in aged people. The AD's patients show progressive memory retardation and cognition disturbance. The pathology of this disease is neuronal loss in the cerebral cortex accompanied by massive accumulation of amyloid fibril forming senile plaque and hyperphosphorylated tua protein forming neurofibrillary tangle (NFT) [82]. The amyloid fibril is mainly composed of $\beta$-amyloid $(\mathrm{A} \beta)$ peptide, the 40 and 42 amino acid form (A $\beta 40$ and $A \beta 42)$, which is derived from proteolytic cleavage from the amyloid precursor protein (APP) by $\beta$ - and $\gamma$-secretase enzyme activity [83-85]. Accumulation of $\mathrm{A} \beta$ plaque, mainly $\mathrm{A} \beta 42$, in the brain parenchyma is the initiation of AD pathogenesis and it leads to the formation of NFT which enhances degeneration of neurons [84]. Presenilin 1(PS1) and presenilin 2 (PS2) genes encode the major component of $\gamma$-secretase which mutated in autosomal-dominant familial Alzheimer's disease [86]. Mutation in the PS1, PS2, and APP genes is reported the most of familial early-onset cases of AD with high level of $A \beta 42$ production and greater fibrillary amyloid deposits [86]. Recently, iPSCs from fibroblasts of FAD with the PS1 mutation A246E and the PS2 mutation N141I were established by retrovirus transduction with the five factors Oct4, SOX2, Klf4, LIN28, and NANOG [87]. All PS1 and PS2 iPSC clones demonstrated typical characteristics of pluripotent cell when compared with both normal 201B7 iPSC line [15] and the sporadic PD-derived iPSC line which was reprogrammed by the original methods [15]. Both PS1 and PS2 lines were induced to differentiate into neurons. Comparing with a normal control, they showed no difference of the efficiency to generate neurons; however, the ratio of $\mathrm{A} \beta 42$ to $\mathrm{A} \beta 40$ was significantly elevated in PS1 and PS2 iPSCs-derived neurons. Surprisingly, neither abnormal tau protein accumulation nor tangle formation was detected in FAD-derived neurons. This may result from the short culture period ( 2 weeks) for tauopathy formation. Not only produced the $\mathrm{A} \beta$ which is the pathological hallmark of $\mathrm{AD}$, FAD-derived neurons also responded to $\gamma$-secretase inhibitor (compound $\mathrm{E}$ ) and $\gamma$-secretase modulator (compound $\mathrm{W}$ ) in order to decrease the ratio of $\mathrm{A} \beta 42$ to $\mathrm{A} \beta 40$. These data highlighted that both PS1 and PS2 iPSC-derived neurons respond to drug treatment as expected and could be useful for a novel drug screening for AD treatment [87]. Nevertheless, neural specific differentiation protocol and long-term culture methods of mature neurons are necessary to enhance dominant disease pathologies.

\section{The Perspectives of Disease Modeling by Human Induced Pluripotent Cells}

One of the most interesting aspects of iPSC technology is the possibility to develop autologous cells for cell replacement therapy. The patient-specific pluripotent cells could be differentiated into desired cell types in the unlimited cell number manner which, ultimately, could be transplanted into the patient's own body. However, techniques of reprogramming somatic cells are necessary to be nonviral, nononcogenic, and nongenetic modification. Moreover, diseases which are related to imprinting genes and epigenetic anomaly, such as FX [44], AS [65], and PWS [48, 65] are difficult to be completely reprogrammed and reset their epigenetic memory [32], which means iPSC technology needs further development in order to overcome these issues. Importantly, the differentiation protocols of desired cell types must be robust and efficient in order to produce high purified specific cell types. For these reasons, the use of iPSCs for cell replacement therapy is not yet ready for clinical applications at this moment [88].

The other applications of iPSCs are the generation of pluripotent cells from developmental or degenerative disorder patients for disease modeling and drug discovery. According to the lack of ideal animal models and inaccessibility to biopsy brain samples from live patients, generation of human pluripotent stem cells has opened an opportunity to investigate disease development in vitro [31]. Ultimately, if iPSCs could be generated in individualized manner, the most effective drug for each patient could be validated $[33,89]$. To date, there are 4 technical challenges if we want to use iPSCs as a disease model. Firstly, the transgenefree iPSCs should be produced in order to minimize or eliminate genetic alterations in the derived iPSC lines. It has been reported that the gene expression features of factor-free PD-iPSCs were closely similar to hESCs [36]. There are many other factor-free methods available for iPSC establishment, such as episomal vector [90, 91], syntheticmodified mRNAs [92], recombinant proteins [93, 94], or miRNA $[95,96]$. Secondly, the solid differentiation protocols of iPSCs into disease-specific cell types must be robust and efficient $[35,87]$. The production of specific cell type in a clinical scale is very difficult, resulting from a short time span of cells in culture conditions. Moreover, specific cellsorting methods have to be developed for purifying only 
disease-related cell types for further investigation. Thirdly, the in vitro disease-relevant phenotypes must be formed. The most important feature for disease-modeling system is the appearance of disease phenotype; however, some of the neural cells derived from patient iPSCs do not show clinical disease phenotypes after the differentiation $[36,44,69]$, in particular iPSCs derived from neurodegenerative diseases with long latency, such as Parkinson's and Huntington diseases. The possibility to overcome this challenge would be the attempt to accelerate the appearance of pathological phenotypes by the exposure of disease stimulators effects, such as oxidative stressors, hydrogen peroxide, or MG-132 $[35,36,87]$. Lastly, the disease-relevant phenotypes could be generated by human-animal chimeras. Some diseases may not be practical to in vitro model since the pathological onset appears in elderly patients with complex pathophysiology. Chimeras provide long-term access to complex and changing environmental context for hiPSCs. Many types of lateonset diseases, such as Alzheimer's and Parkinson's diseases, are multifactorial disorders, caused by both genetic and environmental factors. These chimeric animals will provide the in vivo model for long-term modeling in various types of environmental factors which will provide the close systems to human diseases $[97,98]$, such as triple knockout of amyloid precursor protein (APP), APLP1, and APLP2 chimeric mice which show a mixed population of triple knockout APP neuron in WT back ground brain [99]. In conclusion, the iPSC technology is the powerful technique which allows scientists to investigate the neurological disease development and screen pharmaceutical compounds. Several diseases of the nervous system remain to develop disease-specific iPSCs. In the near future, iPSC technology will facilitate stem cell biologists and neuroscientists to unravel disease mechanisms and discover the new therapeutic approaches for neurological disorders.

\section{Conflict of Interests}

The authors declare no conflict of interests.

\section{Acknowledgments}

The authors apologize to those whose works are not cited because of space constraints. This work was supported by The Royal Golden Jubilee Ph.D. Program of Thailand Research Fund and Suranaree University of Technology.

\section{References}

[1] S. Oddo, A. Caccamo, J. D. Shepherd et al., "Triple-transgenic model of Alzheimer's Disease with plaques and tangles: intracellular A $\beta$ and synaptic dysfunction," Neuron, vol. 39, no. 3, pp. 409-421, 2003.

[2] L. E. Olson, R. J. Roper, L. L. Baxter, E. J. Carlson, C. J. Epstein, and R. H. Reeves, "Down syndrome mouse models Ts65Dn, Ts1Cje, and Ms1Cje/Ts65Dn exhibit variable severity of cerebellar phenotypes," Developmental Dynamics, vol. 230, no. 3, pp. 581-589, 2004.
[3] M. S. Kwon, Y. J. Seo, J. K. Lee et al., "The repeated immobilization stress increases IL- $1 \beta$ immunoreactivities in only neuron, but not astrocyte or microglia in hippocampal CA1 region, striatum and paraventricular nucleus," Neuroscience Letters, vol. 430, no. 3, pp. 258-263, 2008.

[4] J. A. Thomson, "Embryonic stem cell lines derived from human blastocysts," Science, vol. 282, no. 5391, pp. 11451147, 1998.

[5] M. Schuldiner, O. Yanuka, J. Itskovitz-Eldor, D. A. Melton, and N. Benvenisty, "Effects of eight growth factors on the differentiation of cells derived from human embryonic stem cells," Proceedings of the National Academy of Sciences of the United States of America, vol. 97, no. 21, pp. 11307-11312, 2000.

[6] P. Menendez, L. Wang, and M. Bhatia, "Genetic manipulation of human embryonic stem cells: a system to study early human development and potential therapeutic applications," Current Gene Therapy, vol. 5, no. 4, pp. 375-385, 2005.

[7] I. F. Ben-Nun and N. Benvenisty, "Human embryonic stem cells as a cellular model for human disorders," Molecular and Cellular Endocrinology, vol. 252, no. 1-2, pp. 154-159, 2006.

[8] K. H. S. Campbell, P. Fisher, W. C. Chen et al., "Somatic cell nuclear transfer: past, present and future perspectives," Theriogenology, vol. 68, no. 1, pp. S214-S231, 2007.

[9] D. Ben-Yosef, M. Malcov, and R. Eiges, "PGD-derived human embryonic stem cell lines as a powerful tool for the study of human genetic disorders," Molecular and Cellular Endocrinology, vol. 282, no. 1-2, pp. 153-158, 2008.

[10] G. S. Lee, H. S. Kim, S. H. Hyun et al., "Improved developmental competence of cloned porcine embryos with different energy supplements and chemical activation," Molecular Reproduction and Development, vol. 66, no. 1, pp. 17-23, 2003.

[11] E. Einsiedel, S. Premji, R. Geransar, N. C. Orton, T. Thavaratnam, and L. K. Bennett, "Diversity in public views toward stem cell sources and policies," Stem Cell Reviews and Reports, vol. 5, no. 2, pp. 102-107, 2009.

[12] A. J. French, S. H. Wood, and A. O. Trounson, "Human therapeutic cloning (NTSC)," Stem Cell Reviews, vol. 2, no. 4, pp. 265-276, 2006.

[13] M. J. Reiss, "Ethical dimensions of therapeutic human cloning," Journal of Biotechnology, vol. 98, no. 1, pp. 61-70, 2002.

[14] K. Takahashi and S. Yamanaka, "Induction of pluripotent stem cells from mouse embryonic and adult fibroblast cultures by defined factors," Cell, vol. 126, no. 4, pp. 663-676, 2006.

[15] K. Takahashi, K. Tanabe, M. Ohnuki et al., "Induction of pluripotent stem cells from adult human fibroblasts by defined factors," Cell, vol. 131, no. 5, pp. 861-872, 2007.

[16] K. Takahashi, K. Okita, M. Nakagawa, and S. Yamanaka, "Induction of pluripotent stem cells from fibroblast cultures," Nature Protocols, vol. 2, no. 12, pp. 3081-3089, 2007.

[17] I. Griffiths, M. Klugmann, T. Anderson et al., "Axonal swellings and degeneration in mice lacking the major proteolipid of myelin," Science, vol. 280, no. 5369, pp. 1610-1613, 1998.

[18] L. M. Hoffman and M. K. Carpenter, "Characterization and culture of human embryonic stem cells," Nature Biotechnology, vol. 23, no. 6, pp. 699-708, 2005.

[19] J. K. Henderson, J. S. Draper, H. S. Baillie et al., "Preimplantation human embryos and embryonic stem cells show comparable expression of stage-specific embryonic antigens," Stem Cells, vol. 20, no. 4, pp. 329-337, 2002. 
[20] D. C. Hay, L. Sutherland, J. Clark, and T. Burdon, "Oct4 knockdown induces similar patterns of endoderm and trophoblast differentiation markers in human and mouse embryonic stem cells," Stem Cells, vol. 22, no. 2, pp. 225-235, 2004.

[21] H. Darr, Y. Mayshar, and N. Benvenisty, "Overexpression of NANOG in human ES cells enables feeder-free growth while inducing primitive ectoderm features," Development, vol. 133, no. 6, pp. 1193-1201, 2006.

[22] H. Fong, K. A. Hohenstein, and P. J. Donovan, "Regulation of self-renewal and pluripotency by Sox2 in human embryonic stem cells," Stem Cells, vol. 26, no. 8, pp. 1931-1938, 2008.

[23] O. Kopper, O. Giladi, T. Golan-Lev, and N. Benvenisty, "Characterization of gastrulation-stage progenitor cells and their inhibitory crosstalk in human embryoid bodies," Stem Cells, vol. 28, no. 1, pp. 75-83, 2010.

[24] M. Tzukerman, T. Rosenberg, Y. Ravel, I. Reiter, R. Coleman, and K. Skorecki, "An experimental platform for studying growth and invasiveness of tumor cells within teratomas derived from human embryonic stem cells," Proceedings of the National Academy of Sciences of the United States of America, vol. 100, no. 23, pp. 13507-13512, 2003.

[25] M. W. Lensch, T. M. Schlaeger, L. I. Zon, and G. Q. Daley, "Teratoma formation assays with human embryonic stem cells: a rationale for one type of human-animal chimera," Cell Stem Cell, vol. 1, no. 3, pp. 253-258, 2007.

[26] J. B. Kim, H. Zaehres, G. Wu et al., "Pluripotent stem cells induced from adult neural stem cells by reprogramming with two factors," Nature, vol. 454, no. 7204, pp. 646-650, 2008.

[27] D. M. Gupta, N. J. Panetta, N. Sun et al., "Rapid and efficient feeder-free generation of human adipose stromal cell-derived induced pluripotent stem cells (hASC-iPSCs)," Journal of the American College of Surgeons, vol. 209, no. 3, supplement 1, pp. S87-S87, 2009.

[28] A. Giorgetti, N. Montserrat, T. Aasen et al., "Generation of induced pluripotent stem cells from human cord blood using OCT4 and SOX2," Cell Stem Cell, vol. 5, no. 4, pp. 353-357, 2009.

[29] M. E. Brown, E. Rondon, D. Rajesh et al., "Derivation of induced pluripotent stem cells from human peripheral blood T lymphocytes," PLoS One, vol. 5, no. 6, Article ID e11373, 2010.

[30] T. Seki, S. Yuasa, M. Oda et al., "Generation of induced pluripotent stem cells from human terminally differentiated circulating T cells," Cell Stem Cell, vol. 7, no. 1, pp. 11-14, 2010.

[31] M. C. N. Marchetto, C. Carromeu, A. Acab et al., "A model for neural development and treatment of rett syndrome using human induced pluripotent stem cells," Cell, vol. 143, no. 4, pp. 527-539, 2010.

[32] K. Kim, A. Doi, B. Wen et al., "Epigenetic memory in induced pluripotent stem cells," Nature, vol. 467, no. 7313, pp. 285 290, 2010.

[33] V. B. Mattis and C. N. Svendsen, "Induced pluripotent stem cells: a new revolution for clinical neurology?" The Lancet Neurology, vol. 10, no. 4, pp. 383-394, 2011.

[34] A. D. Ebert, J. Yu, F. F. Rose et al., "Induced pluripotent stem cells from a spinal muscular atrophy patient," Nature, vol. 457, no. 7227, pp. 277-280, 2009.

[35] H. A. Nguyen, B. Byers, B. Cord et al., "LRRK2 mutant iPSCderived DA neurons demonstrate increased susceptibility to oxidative stress," Cell Stem Cell, vol. 8, no. 3, pp. 267-280, 2011.
[36] F. Soldner, D. Hockemeyer, C. Beard et al., "Parkinson's disease patient-derived induced pluripotent stem cells free of viral reprogramming factors," Cell, vol. 136, no. 5, pp. 964977, 2009.

[37] N. Zhang, M. C. An, D. Montoro, and L. M. Ellerby, "Characterization of human Huntington's disease cell model from induced pluripotent stem cells," PLoS Currents, vol. 2, p. RRN1193, 2010.

[38] J. T. Dimos, K. T. Rodolfa, K. K. Niakan et al., "Induced pluripotent stem cells generated from patients with ALS can be differentiated into motor neurons," Science, vol. 321, no. 5893, pp. 1218-1221, 2008.

[39] R. J. Hagerman, P. McBogg, and P. J. Hagerman, "The fragile $\mathrm{X}$ syndrome: history, diagnosis, and treatment," Journal of Developmental and Behavioral Pediatrics, vol. 4, no. 2, pp. 122-130, 1983.

[40] D. C. Crawford, J. M. Acuña, and S. L. Sherman, "FMR1 and the fragile $\mathrm{X}$ syndrome: human genome epidemiology review," Genetics in Medicine, vol. 3, no. 5, pp. 359-371, 2001.

[41] C. E. Pearson, K. N. Edamura, and J. D. Cleary, "Repeat instability: mechanisms of dynamic mutations," Nature Reviews Genetics, vol. 6, no. 10, pp. 729-742, 2005.

[42] S. A. Irwin, M. Idupulapati, M. E. Gilbert et al., "Dendritic spine and dendritic field characteristics of layer $\mathrm{V}$ pyramidal neurons in the visual cortex of fragile-X knockout mice," The American Journal of Medical Genetics, vol. 111, no. 2, pp. 140146, 2002.

[43] S. A. Irwin, B. Patel, M. Idupulapati et al., "Abnormal dendritic spine characteristics in the temporal and visual cortices of patients with fragile-X syndrome: a quantitative examination," The American Journal of Medical Genetics, vol. 98 , no. 2, pp. 161-167, 2001.

[44] A. Urbach, O. Bar-Nur, G. Q. Daley, and N. Benvenisty, "Differential modeling of fragile $\mathrm{X}$ syndrome by human embryonic stem cells and induced pluripotent stem cells," Cell Stem Cell, vol. 6, no. 5, pp. 407-411, 2010.

[45] S. J. Chamberlain and M. Lalande, "Neurodevelopmental disorders involving genomic imprinting at human chromosome 15q11-q13," Neurobiology of Disease, vol. 39, no. 1, pp. 13-20, 2010.

[46] K. Buiting, "Prader-Willi syndrome and Angelman syndrome," The American Journal of Medical Genetics, Part C, vol. 154, no. 3, pp. 365-376, 2010.

[47] M. Runte, A. Hüttenhofer, S. Groß, M. Kiefmann, B. Horsthemke, and K. Buiting, "The IC-SNURF-SNRPN transcript serves as a host for multiple small nucleolar RNA species and as an antisense RNA for UBE3A," Human Molecular Genetics, vol. 10, no. 23, pp. 2687-2700, 2001.

[48] J. Yang, J. Cai, Y. Zhang et al., "Induced pluripotent stem cells can be used to model the genomic imprinting disorder Prader-Willi syndrome," Journal of Biological Chemistry, vol. 285, no. 51, pp. 40303-40311, 2010.

[49] C. A. Tamminga, "Diseases of the mind and brain," The American Journal of Psychiatry, vol. 159, no. 7, p. 1103, 2002.

[50] E. C. Azmitia, "Neuronal instability: implications for Rett's syndrome," Brain and Development, vol. 23, no. 1, pp. S1S10, 2001.

[51] A. Blumenfeld, S. A. Slaugenhaupt, F. B. Axelrod et al., "Localization of the gene for familial dysautonomia on chromosome 9 and definition of DNA markers for genetic diagnosis," Nature Genetics, vol. 4, no. 2, pp. 160-164, 1993.

[52] S. A. Slaugenhaupt, A. Blumenfeld, S. P. Gill et al., "Tissuespecific expression of a splicing mutation in the IKBKAP 
gene causes familial dysautonomia," The American Journal of Human Genetics, vol. 68, no. 3, pp. 598-605, 2001.

[53] P. Close, N. Hawkes, I. Cornez et al., "Transcription impairment and cell migration defects in elongator-depleted cells: implication for familial dysautonomia," Molecular Cell, vol. 22, no. 4, pp. 521-531, 2006.

[54] G. Lee, E. P. Papapetrou, H. Kim et al., "Modelling pathogenesis and treatment of familial dysautonomia using patientspecific iPSCs," Nature, vol. 461, no. 7262, pp. 402-406, 2009.

[55] S. L. Anderson, J. Qiu, and B. Y. Rubin, "Tocotrienols induce IKBKAP expression: a possible therapy for familial dysautonomia," Biochemical and Biophysical Research Communications, vol. 306, no. 1, pp. 303-309, 2003.

[56] G. G. V. Simson, J. D. Goldberg, L. M. Rolnitzky et al., "Kinetin in familial dysautonomia carriers: implications for a new therapeutic strategy targeting mRNA splicing," Pediatric Research, vol. 65, no. 3, pp. 341-346, 2009.

[57] A. Dürr, M. Cossee, Y. Agid et al., "Clinical and genetic abnormalities in patients with Friedreich's ataxia," The New England Journal of Medicine, vol. 335, no. 16, pp. 1169-1175, 1996.

[58] M. Voncken, P. Ioannou, and M. B. Delatycki, "Friedreich ataxia-update on pathogenesis and possible therapies," Neurogenetics, vol. 5, no. 1, pp. 1-8, 2004.

[59] S. Al-Mahdawi, R. M. Pinto, P. Ruddle, C. Carroll, Z. Webster, and M. Pook, "GAA repeat instability in Friedreich ataxia YAC transgenic mice," Genomics, vol. 84, no. 2, pp. 301-310, 2004.

[60] S. Al-Mahdawi, R. M. Pinto, D. Varshney et al., "GAA repeat expansion mutation mouse models of Friedreich ataxia exhibit oxidative stress leading to progressive neuronal and cardiac pathology," Genomics, vol. 88, no. 5, pp. 580-590, 2006.

[61] S. Ku, E. Soragni, E. Campau et al., "Friedreich's ataxia induced pluripotent stem cells model intergenerational GAATTC triplet repeat instability," Cell Stem Cell, vol. 7, no. 5, pp. 631-637, 2010.

[62] A. M. Mabb, M. C. Judson, M. J. Zylka, and B. D. Philpot, "Angelman syndrome: insights into genomic imprinting and neurodevelopmental phenotypes," Trends in Neurosciences, vol. 34, no. 6, pp. 293-303, 2011.

[63] C. A. Williams, "The behavioral phenotype of the Angelman syndrome," The American Journal of Medical Genetics, Part C, vol. 154, no. 4, pp. 432-437, 2010.

[64] Y. H. Jiang, T. F. Tsai, J. Bressler, and A. L. Beaudet, "Imprinting in angelman and Prader-Willi syndromes," Current Opinion in Genetics and Development, vol. 8, no. 3, pp. 334-342, 1998.

[65] S. J. Chamberlain, P. F. Chen, K. Y. Ng et al., "Induced pluripotent stem cell models of the genomic imprinting disorders Angelman and Prader-Willi syndromes," Proceedings of the National Academy of Sciences of the United States of America, vol. 107, no. 41, pp. 17668-17673, 2010.

[66] C. Rougeulle, H. Glatt, and M. Lalande, "The Angelman syndrome candidate gene, UBE3A/E6-AP, is imprinted in brain," Nature Genetics, vol. 17, no. 1, pp. 14-15, 1997.

[67] C. Rougeulle, C. Cardoso, M. Fontés, L. Colleaux, and M. Lalande, "An imprinted antisense RNA overlaps UBE3A and a second maternally expressed transcript," Nature Genetics, vol. 19, no. 1, pp. 15-16, 1998.

[68] S. Hussey, C. M. Yates, J. E. Christie, R. A. Elton, and A. Gordon, "Alzheimer-type dementia and Down's syndrome: solubility of neurofibrillary tangles is related to duration of dementia," Journal of Neurology Neurosurgery and Psychiatry, vol. 50, no. 6, pp. 823-824, 1987.

[69] I. H. Park, N. Arora, H. Huo et al., "Disease-specific induced pluripotent stem cells," Cell, vol. 134, no. 5, pp. 877-886, 2008.

[70] A. D. Ebert and C. N. Svendsen, "Stem cell model of spinal muscular atrophy," Archives of Neurology, vol. 67, no. 6, pp. 665-669, 2010.

[71] C. L. Lorson, H. Rindt, and M. Shababi, "Spinal muscular atrophy: mechanisms and therapeutic strategies," Human Molecular Genetics, vol. 19, no. 1, Article ID ddq147, pp. R111-R118, 2010.

[72] M. Feldkötter, V. Schwarzer, R. Wirth, T. F. Wienker, and B. Wirth, "Quantitative analyses of SMN1 and SMN2 based on real-time lightcycler PCR: fast and highly reliable carrier testing and prediction of severity of spinal muscular atrophy," The American Journal of Human Genetics, vol. 70, no. 2, pp. 358-368, 2002.

[73] S. Kato, "Amyotrophic lateral sclerosis models and human neuropathology: similarities and differences," Acta Neuropathologica, vol. 115, no. 1, pp. 97-114, 2008.

[74] F. Gros-Louis, C. Gaspar, and G. A. Rouleau, "Genetics of familial and sporadic amyotrophic lateral sclerosis," Biochimica et Biophysica Acta, vol. 1762, no. 11-12, pp. 956-972, 2006.

[75] D. R. Rosen, T. Siddique, D. Patterson et al., "Mutations in $\mathrm{Cu} / \mathrm{Zn}$ superoxide dismutase gene are associated with familial amyotrophic lateral sclerosis," Nature, vol. 362, no. 6415, pp. 59-62, 1993.

[76] E. Cattaneo, D. Rigamonti, D. Goffredo, C. Zuccato, F. Squitieri, and S. Sipione, "Loss of normal huntingtin function: new developments in Huntington's disease research," Trends in Neurosciences, vol. 24, no. 3, pp. 182-188, 2001.

[77] C. A. Ross and R. L. Margolis, "Huntington's disease," Clinical Neuroscience Research, vol. 1, no. 1-2, pp. 142-152, 2001.

[78] C. Laowtammathron, E. C. H. Cheng, P. H. Cheng et al., "Monkey hybrid stem cells develop cellular features of Huntington's disease," BMC Cell Biology, vol. 11, article 12, 2010.

[79] J. M. Shulman, P. L. De Jager, and M. B. Feany, "Parkinson's disease: genetics and pathogenesis," Annual Review of Pathology, vol. 6, no. 1, pp. 193-222, 2011.

[80] F. J. S. Lee and F. Liu, "Genetic factors involved in the pathogenesis of Parkinson's disease," Brain Research Reviews, vol. 58, no. 2, pp. 354-364, 2008.

[81] J. N. Caviness, L. Lue, C. H. Adler, and D. G. Walker, "Parkinson's disease dementia and potential therapeutic strategies," CNS Neuroscience \& Therapeutics, vol. 17, no. 1, pp. 32-44, 2011.

[82] C. Turkington and D. Mitchell, The Encyclopedia of Alzheimaer's Disease, Infobase Publishing, New York, NY, USA, 2nd edition, 2010.

[83] A. Gonzalo-Ruiz, J. M. Sanz, J. Arévalo, C. Geula, and P. Gonzalo, "Amyloid beta peptide-induced cholinergic fibres loss in the cerebral cortex of the rat is modified by diet high in lipids and by age," Journal of Chemical Neuroanatomy, vol. 29, no. 1, pp. 31-48, 2005.

[84] J. Hardy and D. J. Selkoe, "The amyloid hypothesis of Alzheimer's disease: progress and problems on the road to therapeutics," Science of Aging Knowledge Environment, vol. 297, no. 5580, pp. 353-356, 2002.

[85] S. Kar and R. Quirion, "Amyloid [beta] peptides and central cholinergic neurons: functional interrelationship and relevance to Alzheimer's disease pathology," in Brain Research, K. K. Laurent Descarries and S. Mircea, Eds., pp. 261-274, Elsevier, New York, NY, USA, 2004. 
[86] D. Scheuner, C. Eckman, M. Jensen et al., "Secreted amyloid $\beta$-protein similar to that in the senile plaques of Alzheimer's disease is increased in vivo by the presenilin 1 and 2 and APP mutations linked to familial Alzheimer's disease," Nature Medicine, vol. 2, no. 8, pp. 864-870, 1996.

[87] T. Yagi, D. Ito, Y. Okada et al., "Modeling familial Alzheimer's disease with induced pluripotent stem cells," Human Molecular Genetics, vol. 20, no. 23, pp. 4530-4539, 2011.

[88] K. Saha and R. Jaenisch, "Technical challenges in using human induced pluripotent stem cells to model disease," Cell Stem Cell, vol. 5, no. 6, pp. 584-595, 2009.

[89] J. Ellis and M. Bhatia, "iPSC technology: platform for drug discovery," Clinical Pharmacology \& Therapeutics, vol. 89, no. 5, pp. 639-641, 2011.

[90] Y. Junying, H. Kejin, S. O. Kim et al., "Human induced pluripotent stem cells free of vector and transgene sequences," Science, vol. 324, no. 5928, pp. 797-801, 2009.

[91] B.-K. Chou, P. Mali, X. Huang et al., "Efficient human iPS cell derivation by a non-integrating plasmid from blood cells with unique epigenetic and gene expression signatures," Cell Research, vol. 21, no. 3, pp. 518-529, 2011.

[92] L. Warren, P. D. Manos, T. Ahfeldt et al., "Highly efficient reprogramming to pluripotency and directed differentiation of human cells with synthetic modified mRNA," Cell Stem Cell, vol. 7, no. 5, pp. 618-630, 2010.

[93] D. Kim, C. H. Kim, J. I. Moon et al., "Generation of human induced pluripotent stem cells by direct delivery of reprogramming proteins," Cell Stem Cell, vol. 4, no. 6, pp. 472-476, 2009.

[94] H. Zhou, S. Wu, J. Y. Joo et al., "Generation of induced pluripotent stem cells using recombinant proteins," Cell Stem Cell, vol. 4, no. 5, pp. 381-384, 2009.

[95] F. Anokye-Danso, C. M. Trivedi, D. Juhr et al., "Highly efficient miRNA-mediated reprogramming of mouse and human somatic cells to pluripotency," Cell Stem Cell, vol. 8, no. 4, pp. 376-388, 2011.

[96] C.-S. Yang, Z. Li, and T. M. Rana, "microRNAs modulate iPS cell generation,” RNA, vol. 17, no. 8, pp. 1451-1460, 2011.

[97] R. R. Behringer, "Human-animal chimeras in biomedical research," Cell Stem Cell, vol. 1, no. 3, pp. 259-262, 2007.

[98] L. D. Shultz, F. Ishikawa, and D. L. Greiner, "Humanized mice in translational biomedical research," Nature Reviews Immunology, vol. 7, no. 2, pp. 118-130, 2007.

[99] B. A. Bergmans, S. A. M. Shariati, R. L. P. Habets et al., "Neurons generated from APP/APLP1/APLP2 triple knockout embryonic stem cells behave normally in vitro and in vivo: lack of evidence for a cell autonomous role of the amyloid precursor protein in neuronal differentiation," Stem Cells, vol. 28, no. 3, pp. 399-406, 2010.

[100] A. Colman and O. Dreesen, "Induced pluripotent stem cells and the stability of the differentiated state," EMBO Reports, vol. 10, no. 7, pp. 714-721, 2009.

[101] S. Y. Kim, M.-J. Kim, H. Jung et al., "Comparative proteomic analysis of human somatic cells, induced pluripotent stem cells, and embryonic stem cells," Stem Cells and Development. In press.

[102] S. M. Wu and K. Hochedlinger, "Harnessing the potential of induced pluripotent stem cells for regenerative medicine," Nature Cell Biology, vol. 13, no. 5, pp. 497-505, 2011. 

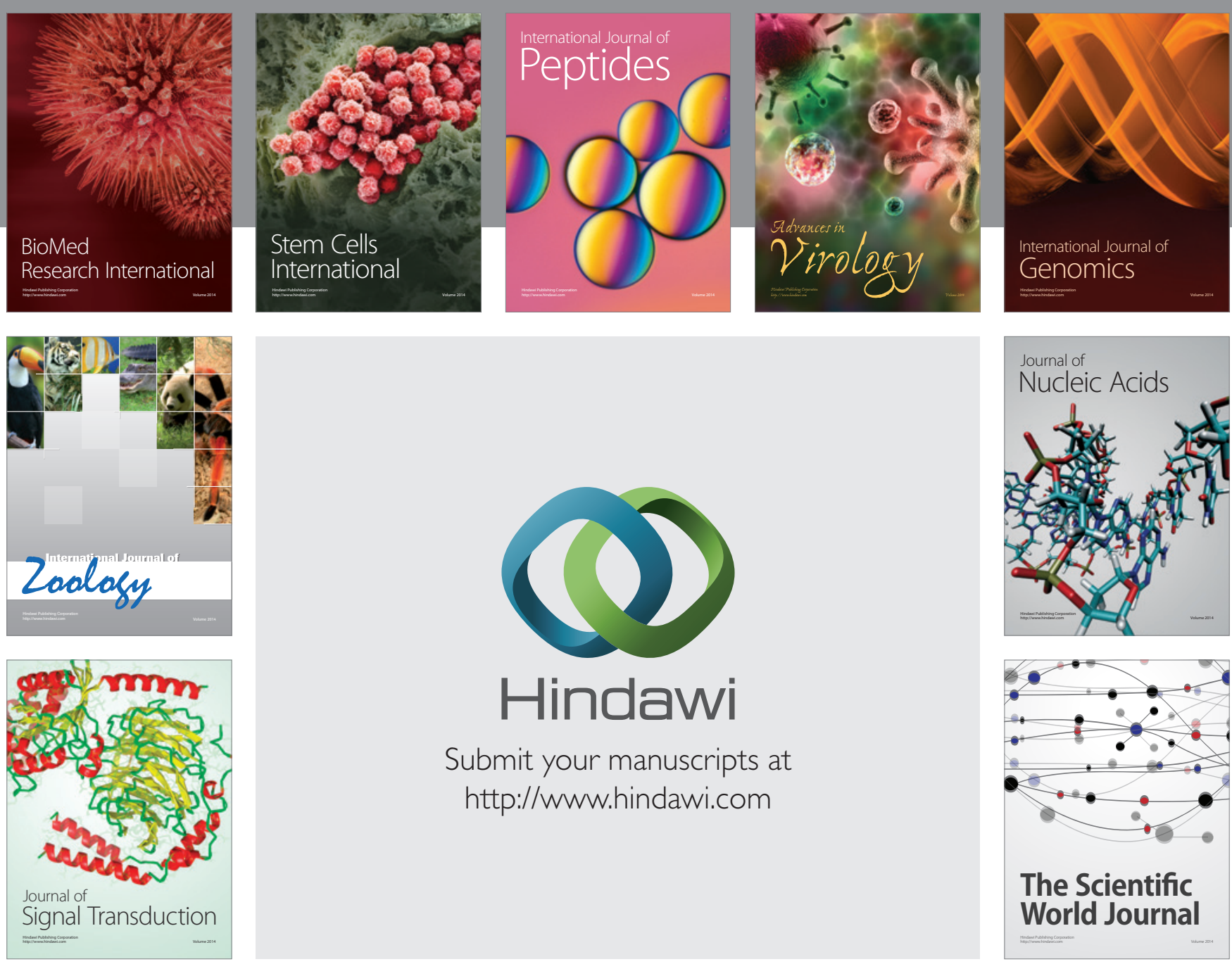

Submit your manuscripts at

http://www.hindawi.com
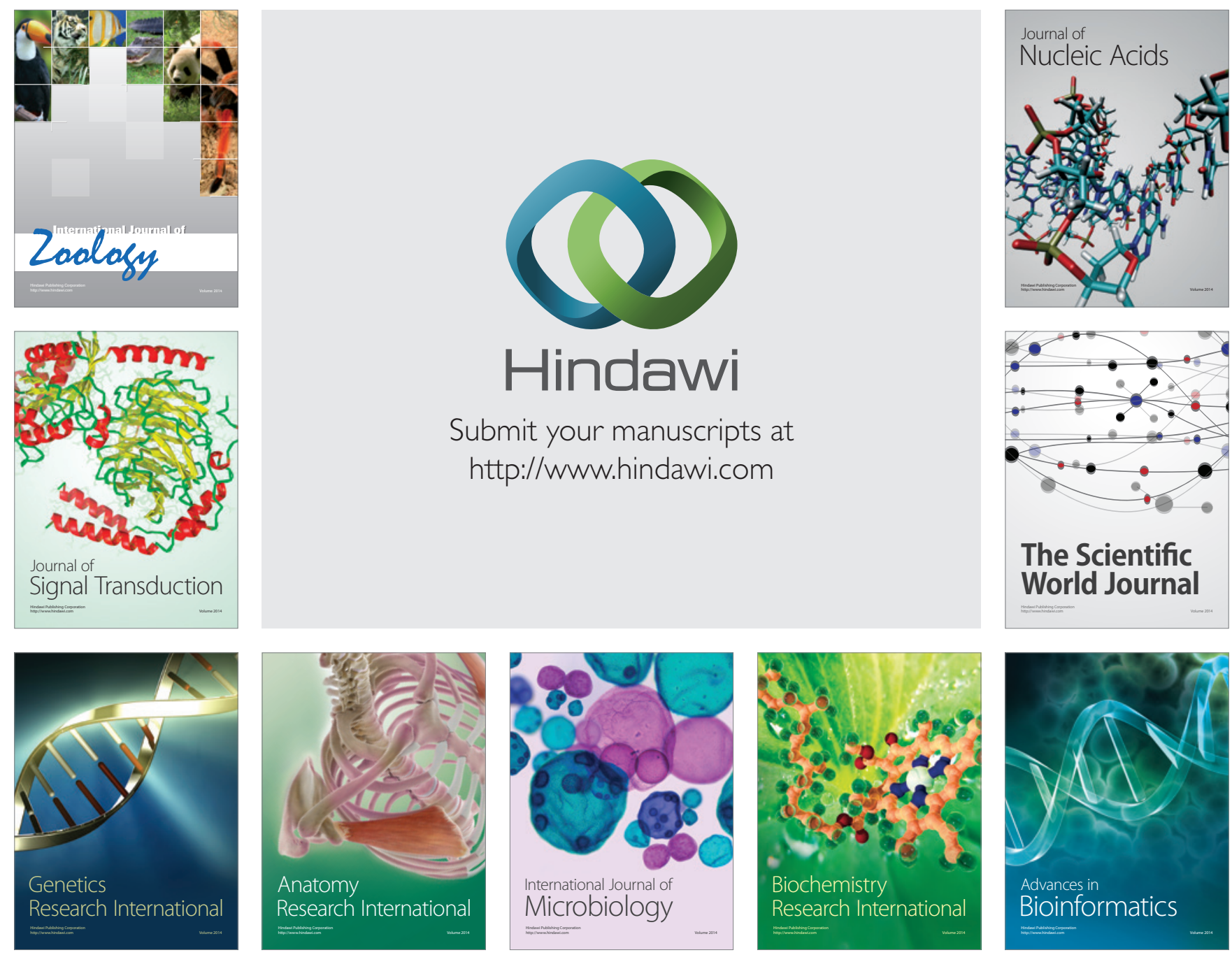

The Scientific World Journal
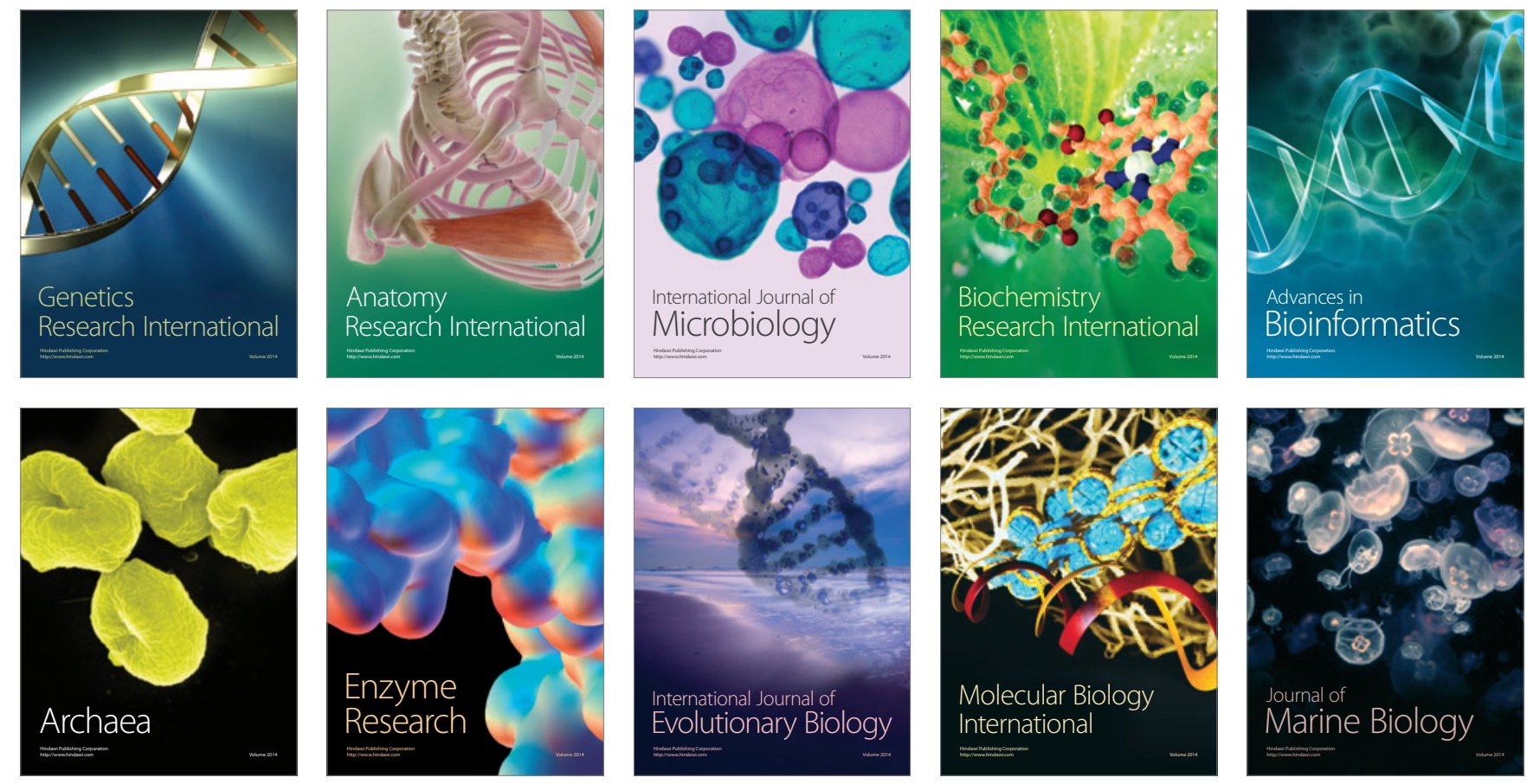